\title{
Diagnostic Performance of One-hour Delta High Sensitive Troponin in Acute Coronary Syndrome
}

\author{
Bahadir Caglar and Suha Serin \\ Department of Emergency Medicine, Balikesir University, Turkey
}

\begin{abstract}
Objective: To determine the impact of an increase in the level of high sensitivite (HS) troponin-l between the time of Emergency Department (ED) arrival and one-hour after arrival (0h-1h), and between the time of ED arrival and three hours after arrival (Oh-3h), upon the diagnostic performance of HS troponin-I to correctly diagnose AMI.

Study Design: Descriptive, analytical study.

Place and Duration of Study: Fethi Sekin City Hospital, Elazig, Turkey from January to June 2019.

Methodology: Patients presenting with chest pain were included in the study. The delta HS Troponin I (Oh-1h) and delta HS Troponin I ( $0 \mathrm{~h}-3 \mathrm{~h}$ ) values of the patients were calculated as percentage changes. These values were statistically compared between those who were diagnosed with acute myocardial infarction (AMI).

Results: The evaluation was performed on 114 patients. Delta HS Troponin I levels were examined, a change of $>20 \%$ was observed in 48 patients $(42.1 \%)$ for the $0-1 \mathrm{hr}$ interval. Of the 20 patients ultimately diagnosed with an $\mathrm{AMI}, 16(80 \%)$ had such a change at this time $(p<0.001)$. For $0-3 \mathrm{hr}$ interval, a change of HS Troponin-I of $>20 \%$ was observed in 52 patients $(45.6 \%)$; and 17 of $20 \mathrm{AMI}$ patients with an AMI were detected by such a change $(p<0.001)$.

Conclusion: Delta HS troponin I value in the first hour was nearly as sensitive for detection of an AMI as was delta HS Troponin I third-hour value.
\end{abstract}

Key Words: Troponin, Myocardial infarction, Acute coronary syndrome.

How to cite this article: Caglar B, Serin S. Diagnostic Performance of One-hour Delta High Sensitive Troponin in Acute Coronary Syndrome. J Coll Physicians Surg Pak 2020; 30(09):906-911.

\section{INTRODUCTION}

Acute myocardial infarction (AMI) takes place on the top among deaths in the world. ${ }^{1}$ The diseases that cause the oxygenation of myocardial cells to deteriorate are acute coronary syndrome (ACS), which can be defined as a whole; sudden cardiac death consists of the subtitles of unstable angina pectoris, ST segment elevated myocardial infarction (STEMI) and non-ST segment elevation myocardial infarction (NSTEMI).

Today, the diagnosis of AMI is made by symptoms, electrocardiography (ECG) and cardiac biomarkers. ${ }^{2,3}$ Cardiac biomarkers are used in patients without ST segment elevation and with ischemic symptoms on ECG. ${ }^{2}$ Troponin T and Troponin I, known as cardiac troponins, are among the most widely used biomarkers. ${ }^{4,5}$ An increase in Troponin I from extra-cardiac tissues has not been reported. ${ }^{2}$

Correspondence to: Dr. Bahadir Caglar, Department of Emergency Medicine, Balikesir University, Turkey

E-mail: mail@bahadircaglar.com

Received: June 25, 2020; Revised: September 17, 2020;

Accepted: September 30, 2020

DOI: https://doi.org/10.29271/jcpsp.2020.09.906
In recent years, highly sensitive (HS) types of troponins are preferred because they respond earlier. When the troponin value exceeds 99 percantil upper referance limit $\left(99^{\text {th }} U R L\right)$, it is an indication that myocard injury has occurred. ${ }^{2}$ If there is an increase or decrease in the troponin value, this is an indication that the damage is acute and may be referred to as AMI. ${ }^{2}$ It is stated in the literature that a $20 \%$ change in serial troponin measurements can be used as an AMI finding ${ }^{2,6,7}$ As for the time of serial troponin measurements, the recommended arrival and 3rd hour troponin value should be seen., This change in troponin, called delta $(\Delta)$, may be undetectable due to the nature of the troponin ascent curve, if sampling the pattern is done too early or too late. ${ }^{2}$

The objective of this study was to compare the changes in troponin values at the $1^{\text {st }}$ hour and $3^{\text {rd }}$ hour, according to the arrival troponin value in terms of diagnostic performance on AMI (delta HS troponin 0-1h/delta HS troponin 0-3h).

\section{METHODOLOGY}

Patients who applied to the Emergency Department with ischemic symptoms suggestive of chest pain or ACS were included in the study. Pregnant women, those under the age of 18 years, those who did not agree to participate in the study, those who applied to the Emergency Room within the last 24 
hours, those having a history of coronary intervention in the last month (cardiac bypass surgery, percutaneous coronary angioplasty), those who were in the haemodialysis programme, those with first troponin value $<18 n g /$ l $\left(<99^{\text {th }} U R L\right)$, ST segment elevation in their ECG, as well as patients who were evaluated by the cardiologist for AMI with the first troponin result were excluded from the study.

The patients in this study were evaluated by emergency physicians blinded to the study design. Blood was drawn 3 times for troponin value from patients whose ECGs were taken at the time of application (0h), $1^{\text {st }}$ hour and $3^{\text {rd }}$ hour. Blood from gel tube (BD vacutainer SST II) studied through chemiluminescent immunoassay two step immunoenzymatic (sandwich) method with ACCESS hsTnl (RefB52699) kit in the Unicel DXI 800 accuTnl (Beckman Coulter, Inc., Fulerton, CA) device in the hospital centre laboratory. The results were obtained through the hospital data processing system. All patients were evaluated by a cardiologist with ECG, blood results, and echocardiography. Patients diagnosed with cardiac pathology were hospitalised. According to the HS troponin I results of the patients, delta HS troponin I (0h-1h), delta HS troponin I (0h-3h) and delta HS troponin I (1h-3h) values were calculated as percentage change. These values were compared statistically between those who were diagnosed with AMI and those who were not.

The study was carried out in accordance with Helsinki Declaration after Ethics Committee sapproval.

Variables in the study were analysed statistically using SPSS version 25.0 (IBM Corporation, Armonk, New York, United States) and PAST 3 (Hammer, Ø., Harper, D.A.T., Ryan, P.D. 2001. Paleontological statistics). Kolmogorov-Smirnov test and Shapiro-Wilk test were used to assess the conformity of the univariate data to normal distribution. In order to assess the multivariate normal distribution, Mardia (Dornik and Hansen omnibus) test was used. Mann-Whitney U-test was used in the comparisons of quantitative data of two independent groups. Friedman's Two-Way test was used in the comparison of two repetitive measurements of the dependent quantitative variables, and Dunn's Test was used for Post Hoc analysis. In the comparison of categorical variables with each other, Pearson Chi-square test was used. The odds ratio was used with $95 \%$ confidence intervals to indicate how many times higher the risk factor is compared with those not possessing a risk factor. In order to show the relationship between the categorisation created by the cut-off value which was calculated based on the variables - and the actual categorisation. The sensitivity, specificity and diagnostic accuracy likelihood odds were analysed and indicated by receiver operating characteristic (ROC) curve analysis. Neural Network (Multilayer Perceptron) was implemented both to determine the most important variable and for estimation as well. Gradient descent was used for optimisation algorithm, hyperbolic tangent was used as hidden layer activation function, and identity was used as output layer activation function. Furthermore, mini-batch method was used for training data selection, and it was set as 100\% training set and $0 \%$ testing set. The quantitative variables were shown as median (25th percentiles (Q1) / 75th percentiles (Q3)), while the categorical variableswere shown asn (\%) in the Tables. The variables were analysed at $95 \%$ confidence level, and the p-value was accepted as significant when it was lower than 0.05 .

\section{RESULTS}

Between January and July 2019, 442 patients applied to the adult Emergency Department with a complaint suitable for the study. Sixty-four of these patients did not agree to participate in the study. Some patients were excluded from the study due to the various reasons as follows: Seven patients were on haemodialysis programme due to chronic renal failure; 9 patients left the emergency service at their own request while the treatment was continuing; 22 patients were diagnosed with STEMI at presentation or during follow-up; and 29 patients were considered AMI by the cardiologist with the first troponin result. The first troponin result of 197 patients was $<18 \mathrm{ng} /$ / $\left(99^{\text {th }} U R L\right)$. The final evaluation was made on 114 patients.

As a result of follow-up and consultations for 114 patients in the Emergency Department, 80 patients (70.2\%) were discharged after their treatment was regulated. Twenty-six patients (22.8\%) were hospitalised in coronary intensive care unit due to cardiac pathology, and $8(7 \%)$ patients in other clinics. Twenty of the patients hospitalised in coronary intensive care had (17.5\%) AMI, three patients had tachycardia, two patients had congestive heartfailure and one patient had pericarditis.

Of the 80 patients included in the study and discharged, 78 were reached by phone 7-11 days after their discharge. After discharge, the ACS and mortality histories of the patients were questioned. No ACS or mortality was detected in the patients reached. Two patients could not be reached due to missing contact information. It was checked through the national death reporting system, and no mortality was detected. There was no statistically significant difference by age and gender between those who were diagnosed with AMl and those who did not (Tablel).

When delta HS troponin I levels were examined in 0-1-hour interval, a change of $>20 \%$ was found in $48(42.1 \%)$ of 114 patients; and 16 of these patients had AMI [ $p<0.001$, odds ratio: 7.8, 2.4-25.1)]. While 52 (45.6\%) patients with delta HS troponin I $>20 \%$ were detected between the range of $0-3$ hour. In the AMI group, it was found to be 17 patients in this range, $[p<0.001$, odds ratio: 9.6 (2.6-34.9, Table I]. Between 1-3 hours, while delta HS troponin I was $>20 \%$ in $45(39.5 \%)$ patients, this rate was found to be 17 patients in the AMI group, $[p<0.001$ odds ratio: 13.4 (3.6-49.2, Tablel).

The cut off values were determined as the most appropriate increase rate in troponin value for AMI diagnosis, as follows: delta HS troponin I $>66.7 \%$ (sensitivity $75.0 \%$, specificity $79.8 \%$, odds ratio: 11.84 ) $0-3 \mathrm{~h}$ delta HS troponin I $>148.3 \%$ (sensitivity $80.0 \%$, specificity $85.1 \%$ odds ratio: 22.86 ) in the range $1-3 \mathrm{~h}$ delta HS troponin I $>47.2 \%$ (sensitivity $80.0 \%$, specificity 89 , odds ratio: 33,59, Table II). 
Table I: Delta HS troponin I and AMI relationship.

\begin{tabular}{|c|c|c|c|c|c|}
\hline & $\begin{array}{c}\text { Total }(n=114) \\
\text { Median (Q1-Q3) }\end{array}$ & $\begin{array}{l}\text { ACS Absent }(n=94) \\
\text { Median (Q1-Q3) }\end{array}$ & $\begin{array}{l}\text { ACS Present }(n=20) \\
\text { Median (Q1-Q3) }\end{array}$ & \multirow[t]{2}{*}{$\mathbf{P}$} \\
\hline & & $\mathrm{n}(\%)$ & $\mathrm{n}(\%)$ & $\mathrm{n}(\%)$ & \\
\hline \multicolumn{2}{|l|}{ Age } & $64(53-74)$ & $63(52-72)$ & $68(56-79)$ & $0.100^{u}$ \\
\hline \multirow{2}{*}{\multicolumn{2}{|c|}{$\begin{array}{l}\text { Sex } \\
\text { Female } \\
\text { Male }\end{array}$}} & $\begin{array}{l}44(38.6) \\
70(61.4)\end{array}$ & $\begin{array}{l}36(38.3) \\
58(61.7)\end{array}$ & $\begin{array}{c}8(40.0) \\
12(60.0)\end{array}$ & $0.887^{p}$ \\
\hline & & $\begin{array}{c}\text { Total }(n=114) \\
n(\%)\end{array}$ & $\begin{array}{c}\text { ACS Absent }(n=94) \\
n(\%)\end{array}$ & $\begin{array}{c}\text { ACS Present }(n=20) \\
n(\%)\end{array}$ & $P$ \\
\hline \multicolumn{6}{|l|}{ delta hs Trop I (0-1)h $\pm 20 \%$} \\
\hline \multicolumn{2}{|l|}{ Non risc } & $66(57.9)$ & $62(66.0)^{\prime \prime}$ & $4(20.0)$ & $<0.001^{p}$ \\
\hline \multicolumn{2}{|l|}{ Risc of ACS } & $48(42.1)$ & $32(34.0)$ & $16(80.0)^{\prime}$ & $7.8(2.4-25.1)^{\text {or }}$ \\
\hline \multicolumn{6}{|l|}{ delta hs Trop I $(0-3) \mathrm{h} \pm 20 \%$} \\
\hline \multicolumn{2}{|l|}{ Non risc } & $62(54.4)$ & $59(62.8)^{\prime \prime}$ & $3(15.0)$ & $<0.001^{p}$ \\
\hline \multicolumn{2}{|l|}{ Risc of ACS } & $52(45.6)$ & $35(37.2)$ & $17(85.0)^{\prime}$ & $9.6(2.6-34.9)$ or \\
\hline \multicolumn{6}{|l|}{ delta hs Trop I (1-3)h $\pm 20 \%$} \\
\hline \multicolumn{2}{|l|}{ Non risc } & $69(60.5)$ & $66(70.2) "$ & $3(15.0)$ & $<0.001^{p}$ \\
\hline \multirow{2}{*}{\multicolumn{2}{|c|}{ Risc of ACS }} & $45(39.5)$ & $28(29.8)$ & $17(85.0)^{\prime}$ & $13.4(3.6-49.2)^{\text {or }}$ \\
\hline & & Median (Q1.-Q3.) & Median (Q1.-Q3.) & Median (Q1.-Q3.) & \\
\hline \multicolumn{2}{|l|}{ delta hs Trop I 1h-0h \% } & $12.3(1.7-91.4)$ & $9.9(1.4-43.0)$ & $130.8(53.5-335.2)$ & $<0.001^{u}$ \\
\hline \multicolumn{2}{|l|}{ delta hs Trop I 3h-0h \% } & $18.2(0.6-200.3)$ & $14.8(0.5-77.7)$ & $820.5(290.5-2056.5)$ & $<0.001^{u}$ \\
\hline \multicolumn{2}{|l|}{ delta hs Trop I 3h-1h \% } & $8.4(-4.3-46.1)$ & $4.1(-5.2-20)$ & $133.5(51.5-347.6)$ & $<0.001^{u}$ \\
\hline \multicolumn{2}{|l|}{ hs Trop I 0h ng/l (A) } & $24.2(8.9-45.1)$ & $22.5(7.1-39.4)$ & $46.3(33.4-85.7)$ & $<0.001^{u}$ \\
\hline \multicolumn{2}{|l|}{ hs Trop I lh ng/l (B) } & $33.1(19.1-90.5)$ & $30.5(12.5-51.5)$ & $153.6(91.6-418.3)$ & $<0.001^{u}$ \\
\hline \multicolumn{2}{|l|}{ hs Trop I 3h ng/l (C) } & $39.1(20.9-142.8)$ & $30.6(14.6-58.1)$ & $387.9(249.1-1059.2)$ & $<0.001^{u}$ \\
\hline \multicolumn{2}{|l|}{$P$ value for intra groups ${ }^{f}$} & $<0.001$ & $<0.001$ & $<0.001$ & \\
\hline \multirow{3}{*}{ Pairwise comparation } & A-B & $<0.001$ & $<0.001$ & 0.027 & \\
\hline & A-C & $<0.001$ & $<0.001$ & $<0.001$ & \\
\hline & B-C & 0.017 & 0.109 & 0.027 & \\
\hline
\end{tabular}

Table II: Cutoff variables determined according to the delta time at HS troponin I.

\begin{tabular}{|l|c|c|c|}
\hline \multirow{2}{*}{} & Delta HS Trop I 1h-0h \% & Delta HS Trop I 3h-0h \% & Delta HS Trop I 3h-1h \% \\
\cline { 2 - 4 } & ( A ) & ( B ) & $>47.2$ \\
\hline Cut off & $>66.7$ & $>148.3$ & $80.0 \%$ \\
\hline Sensitivity & $75.0 \%$ & $80.0 \%$ & $89.4 \%$ \\
\hline Specificity & $79.8 \%$ & $85.1 \%$ & $87.72 \%$ \\
\hline Accuracy rate & $78.95 \%$ & $84.21 \%$ & $0.838 \pm 0.066$ \\
\hline AUC \pm SE. & $0.755 \pm 0.072$ & $0.809 \pm 0.074$ & $<0.001$ \\
\hline P & $<0.001$ & $<0.001$ & P (B-C) $=0.613$ \\
\hline Pairwise comparation for AUC & P (A-B) $=0.291$ & A-C) $=0.323$ & $33.59(0.81)$ \\
\hline Odds Ratio (SE.) & $11.84(0.70)$ & $22.86(0.77)$ & \\
\hline Roc (Receiver Operating Curve) Analysis (Honley\&MC Nell - Youden index J) AUC: Area under the ROC curve SE: Standard Error.
\end{tabular}

Table III: Neural network models for prediction of AMI.

\begin{tabular}{|c|c|c|c|c|c|c|}
\hline \multirow[b]{2}{*}{ Model } & \multicolumn{2}{|c|}{ Variable Importance } & \multirow{2}{*}{$\begin{array}{l}\text { Sample (Holdout) } \\
\text { Training (\%100) }\end{array}$} & \multicolumn{3}{|c|}{ Predicted AMI } \\
\hline & Independent Variable & $\begin{array}{l}\text { Normalized } \\
\text { Importance }\end{array}$ & & Absent & Present & Percent Correct \\
\hline \multirow{3}{*}{1} & Delta hs Trop I (0-1)h $\pm 20 \%$ & $75.4 \%$ & AMI Absent & 94 & 0 & $100.0 \%$ \\
\hline & Delta hs Trop I (0-3)h $\pm 20 \%$ & $72.1 \%$ & AMI Present & 20 & 0 & $0.0 \%$ \\
\hline & Delta hs Trop I (1-3)h $\pm 20 \%$ & $100.0 \%$ & Overall Percent & $100.0 \%$ & $0.0 \%$ & $82.5 \%$ \\
\hline \multirow{3}{*}{2} & Delta Cut off 1h-0h \% & $39.0 \%$ & AMI Absent & 87 & 7 & $92.6 \%$ \\
\hline & Delta Cut off 3h-0h \% & $38.6 \%$ & AMI Present & 5 & 15 & $75.0 \%$ \\
\hline & Delta Cut off 3h-1h \% & $100.0 \%$ & Overall Percent & $80.7 \%$ & $19.3 \%$ & $89.5 \%$ \\
\hline
\end{tabular}


In addition, in the neural network modelling (Model 1) made, based on the change in delta HS troponin I levels of $>20 \%$, none of the patients with AMI could accurately be predicted. However, with a model created according to the cut-off values (Model 2), patients with AMI could be predicted with $75 \%$ accuracy and $89.5 \%$ in total. In both models, normalised importance was found at the maximum delta value between 1-3h (Table III, Figure 1).

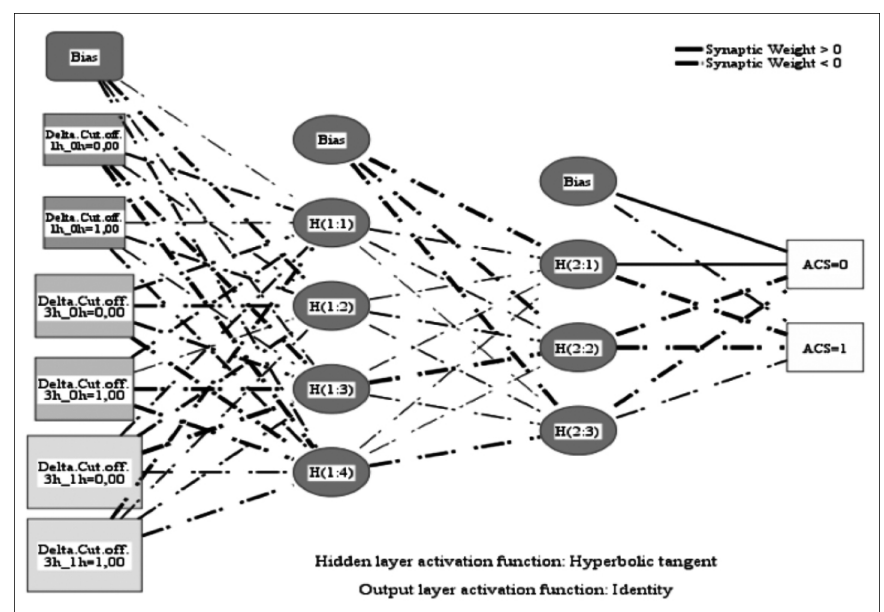

Figure 1: Model 2 of the neural network.

\section{DISCUSSION}

AMI is classified as type 1 , if it develops due to atherosclerotic heart disease; and type 2 , if it develops due to the mismatch between oxygen supply and demand. ${ }^{2}$ The distinction of these two is clinically difficult. While an invasive approach is not required for most patients in type $2 \mathrm{MI}$, an invasive approach is required primarily in type $1 \mathrm{MI}$. At this point, the amount of increase in high sensitivity troponins is important. If the increase in troponin value is excessive, first of all, invasive approach should be considered. ${ }^{9}$

While it is emphasised in the guidelines that at least two serial measurements should be made. ${ }^{8}$ It is stated that troponin measurement intervals can be increased to 3 hours, and up to 6 hours in suspected patients. ${ }^{8}$ However, with the advancing technology and the increasing use of highly sensitive troponin, studies are being conducted to further shorten these periods. ${ }^{10-14}$

Given that cellular ischemia results in cellular dysfunction within 10 minutes of the onset of an AMI, early and accurate diagnosis has a high clinical importance. Accurate diagnosis enables rapid identification of patients who merit an emergency invasive revascularisation procedure, performed with the intention of preserving myocardial function and avoiding subsequent problems such as sudden cardiac death or congestive heart failure. Early identification also could decrease the ED length of stay, and thus improve ED throughput times and efficiency. ${ }^{10}$

Although there are delta troponin studies up to 30 minutes in the literature, 2015 ESC manual, under certain conditions, contains information about rapid discharge regarding 1 hour delta troponin levels. ${ }^{1,15}$ In literature in similar studies, patients below the level of detection value were examined to quickly exclude the diagnosis of AMI. ${ }^{16-20}$ The authors investigated for the faster diagnosis of patients with HS troponin $1>99^{\text {th }}$ URL at presentation.

For the diagnosis of AMI, authors compared the percentage change in HS troponin I at the 1st hour with the percentage change at the 3rd hour (diagnostic performance). In patients diagnosed with AMI determination was done as follows: 16 patients $(80 \%)$ with delta HS troponin I $(0-1 \mathrm{~h})>20 \%$ (p $<0.001$ odds ratio: $7.8(2.4-25.1)$ ), delta HS troponin I (0-3h) $>20 \%$ in 17 patients [ $85 \%, p<0.001$, odds ratio: 9.5 (2.6-34.9)].

However, the highest odds ratio was found as delta HS troponin $\mathrm{I}(1-3 \mathrm{~h})>20 \%$ ( $p<0.001$ odds ratio: 13.3 (3.6-49.2)). The reason of this might be the variation in the perception of symptoms by persons, which can be very subjective. Therefore, the symptom duration of the patients was not explained in this study. Troponin value starts to increase with the onset of symptoms. However, due to troponin kinetics, when sampling too early or late, the delta value may not be detected. ${ }^{2}$

Delta troponin values in the range of $1-3 \mathrm{~h}$ in the presently reported two case groups corresponded to the rising period in the troponin kinetic curve.

According to the cut-off value, the maximum diagnostic accuracy is delta $\mathrm{HS}$ between $1-3$ hours troponin $\mathrm{I}>47.2 \%$ had a sensitivity (80.0\%) and specificity (89.4\%) with AUC of 0.838 \pm 0.066 ( $p<0.001)$. Secondly, delta HS between $0-3$ hours troponin I $>148.3 \%$ with sensitivity of $80.0 \%$, specificity of $85.1 \%$, AUC $0.809 \pm 0.074$ and $p<0.001$. Finally, delta HS troponin $\mathrm{I}>66.7 \%$ has a sensitivity of $75.0 \%$ and specificity of $79.8 \%$ at 0 -1-hour interval, AUC $0.755 \pm 0.072$ ( $p$ $<0.001$ ). In the study conducted by Keller et al. ${ }^{11}$ in patients with HS troponin I level $>99^{\text {th }}$ URL at the time of admission; when the third hour cut-off value was $>250 \%$, they determined the specificity as 99.6, sensitivity 32.6, and PPV 95.8. ${ }^{11}$ Although the diagnostic power was greatest at the delta HS troponin I (1-3h) change; the delta HS troponin I (0-1h) change and the delta HS troponin I (0-3h) changes are very close in terms of diagnostic power. For this reason, one should consider acting earlier, rather than waiting 3 hours for the second troponin sample, as stated in the literature in patients with a high probability of ACS clinically. ${ }^{8}$ At this point, the patient's history, age and risk factors are very important. However, do not forget the other reasons that increase troponin values in low changes or the factors that cause type $2 \mathrm{Ml}^{9}{ }^{9}$ When a statistically neural network study was done, the model established based on $>20 \%$ change in troponin value could not detect any of the patients with AMI. The second model, built on the basis of cut-off values, 
detected $75 \%$ of patients diagnosed with AMI and achieved an accuracy rate of $89.5 \%$ in all patients.

Although the low number of patients with AMI diagnosis is considered as the main limitation, the time between the onset of symptoms and admission to the hospital should be clearly demonstrated in order for such studies to yield better results. The authors did not explore the symptom duration in the study because of possible recall bias.

A relative value was used as the delta troponin value. In studies comparing absolute and relative delta troponin in the literature, although both were effective in the diagnosis of AMI, absolute $u$ was found to be statistically superior. ${ }^{14,21}$ Using relative and absolute delta troponin values together in this study could have shown a more detailed approach.

\section{CONCLUSION}

In AMI, the initiation time of treatment is closely related to mortality and morbidity. The delta HS troponin I 0-1h will be as useful as delta HS troponin I $0-3 \mathrm{~h}$ in diagnosing NSTEMI. It is noted that a change of $>20 \%$ in the HS troponin value specified in the manuals will not be sufficient in future models of artificial neural networks, but the models to be established with a higher rate of change will be effective in confirming the diagnosis of AMI.

\section{ETHICAL APPROVAL:}

This research was carried out after obtaining permission from the Ethics Committee of the Firat University, Elazig, Turkey.

\section{PATIENTS' CONSENT:}

Consents were obtained from all patients.

\section{CONFLICT OF INTEREST:}

Authors declared no conflict of interest.

\section{AUTHORS' CONTRIBUTION:}

BC: Analysis and interpretation of data and critical revision. SS: Study conception and design, acquisition of data and drafting of manuscript.

\section{REFERENCES}

1. Yokoyama H, Higuma T, Endo T, Nishizaki F, Hanada $K$, Yokota T, et al. "30-minute-delta" of high-sensitivity Troponin I improves diagnostic performance in acute myocardial infarction. J Cardiol 2018; 71(2):144-8. doi: 10.1016/j.jjcc.2017.08.003.

2. Thygesen K, Alpert JS, Jaffe AS, Chaitman BR, Bax JJ, Morrow DA, et al. Fourth universal definition of myocardial infarction (2018). J Am Coll Cardiol 2018; 72(18):2231-64. doi: 10.1161/CIR.0000000000000617.

3. Alpert JS, Antman E, Apple F, Armstrong PW, Bassand JP, De Luna $A B$, et al. Myocardial infarction redefined - $A$ consensus document of the joint european society of cardiology/american college of cardiology committee for the redefinition of myocardial infarction. J Am Coll Cardiol 2000; 36(3):959-69. doi: 10.1016/s0735-1097(00)00804-4.

4. Thygesen K, Mair J, Katus H, Plebani M, Venge P, Collinson P, et al. Recommendations for the use of cardiac troponin measurement in acute cardiac care. Eur Heart J 2010; 31(18):2197-204. doi: 10.1093/eurheartj/ehq251.

5. Apple FS, Jaffe AS, Collinson P, Mockel M, Ordonez-Llanos J, Lindahl B, et al. IFCC educational materials on selected analytical and clinical applications of high sensitivity cardiac troponin assays. Clin Biochem 2015; 48(4-5): 201-3. doi: 10.1016/j.clinbiochem.2014.08.021.

6. Apple FS, Murakami MM. Cardiac troponin and creatine kinase MB monitoring during in-hospital myocardial reinfarction. Clin Chem 2005; 51(2):460-3. doi: 10.1373/ clinchem.2004.042887.

7. Thygesen K, Mair J, Giannitsis E, Mueller C, Lindahl B, Blankenberg $\mathrm{S}$, et al. How to use high-sensitivity cardiac troponins in acute cardiac care. Eur Heart J 2012; 33(18):2252-7. doi: 10.1093/eurheartj/ehs154.

8. Amsterdam EA, Wenger NK, Brindis RG, Casey DE Jr, Ganiats TG, Holmes DR Jr, et al. 2014 AHA/ACC guideline for the management of patients with non-ST-elevation acute coronary syndromes: A report of the American college of cardiology/American heart association task force on practice guidelines. J Am Coll Cardiol 2014; 64(24):e139-228. DOI: 10.1016/j.jacc.2014.09.017

9. High-sensitivity cardiac troponin in the evaluation of possible AMI - American college of cardiology [Internet]. Acc.org. [cited 2020 Sep 8]. Available from: http:// www.acc.org/latest-in-cardiology/articles/2018/07/16/09/17/ high-sensitivity-cardiac-troponin-in-the-evaluation-of-possible-ami.

10. Nowak RM, Gandolfo CM, Jacobsen G, Christenson RH, Moyer $M$, Hudson $M$, et al. Ultrarapid rule-out for acute myocardial infarction using the Generation 5 cardiac troponin T assay: Results from the REACTION-US study. Ann Emerg Med 2018; 72(6):654-64. doi: 10.1016/j.anne mergmed.2018.06.021.

11. Keller T, Zeller T, Ojeda F, Tzikas S, Lillpopp L, Sinning C, et al. Serial changes in highly sensitive troponin i assay and early diagnosis of myocardial infarction. JAMA 2011; 306(24): 2684-93. doi: 10.1001/jama.2011.1896.

12. Apple FS, Pearce LA, Smith SW, Kaczmarek JM, Murakami MM. Role of monitoring changes in sensitive cardiac troponin I assay results for early diagnosis of myocardial infarction and prediction of risk of adverse events. Clin Chem 2009; 55(5):930-7. doi: 10.1373/clinchem.2008. 114728.

13. Wildi K, Reichlin T, Twerenbold R, Mäder F, Zellweger $C$, Moehring $B$, et al. Serial changes in high-sensitivity cardiac troponin I in the early diagnosis of acute myocardial infarction. Int J Cardiol 2013; 168(4):4103-10. doi: 10.1016/j.ijcard.2013.07.078.

14. Mueller M, Biener M, Vafaie M, Doerr S, Keller T, Blankenberg $S$, et al. Absolute and relative kinetic changes of high-sensitivity cardiac troponin $\mathrm{T}$ in acute coronary syndrome and in patients with increased troponin in the absence of acute coronary syndrome. Clin Chem 2012; 58(1):209-18. doi: 10.1373/clinchem.2011.171827. 
15. Roffi M, Patrono C, Collet JP, Mueller C, Valgimigli M, Andreotti F, et al. 2015 ESC Guidelines for the management of acute coronary syndromes in patients presenting without persistent ST-segment elevation: Task force for the management of acute coronary syndromes in patients presenting without persistent st-segment elevation of the european society of cardiology (ESC). Eur Heart J 2016; 37(3): 267-315. doi: 10.1093/eurheartj/ehv320.

16. Neumann JT, Sörensen NA, Schwemer T, Ojeda F, Bourry R, Sciacca $V$, et al. Diagnosis of myocardial infarction using a high-sensitivity troponin I 1-hour algorithm. JAMA Cardiol 2016; 1(4):397-404. doi: 10.1001/jamacardio.2016.0695.

17. Jaeger C, Wildi K, Twerenbold R, Reichlin T, Rubini Gimenez $M$, Neuhaus J-D, et al. One-hour rule-in and rule-out of acute myocardial infarction using high-sensitivity cardiac troponin I. Am Heart J 2016; 171(1):92-102.e1-5. doi: 10.1016/j.ahj. 2015.07.022.

18. Rubini Gimenez M, Twerenbold R, Jaeger C, Schindler C,
Puelacher $\mathrm{C}$, Wildi $\mathrm{K}$, et al. One-hour rule-in and rule-out of acute myocardial infarction using high-sensitivity cardiac troponin I. Am J Med 2015; 128(8):861-70.e4. doi: 10.1016/j.amjmed.2015.01.046.

19. Reichlin T, Twerenbold R, Wildi K, Gimenez MR, Bergsma N, Haaf $P$, et al. Prospective validation of a 1-hour algorithm to rule-out and rule-in acute myocardial infarction using a high sensitivity cardiac troponin T assay. CMAJ 2015; 187(8):e243-e252. doi: 10.1503/cmaj.141349.

20. Mueller C, Giannitsis E, Christ M, Ordóñez-Llanos J, DeFilippi C, McCord J, et al. Multicenter evaluation of a 0-hour/1-hour algorithm in the diagnosis of myocardial infarction with high-sensitivity cardiac troponin T. Ann Emerg Med 2016; 68(1): 76-87. doi: 10.1016/j.annemergmed.2015. 11.013.

21. Reichlin T, Irfan A, Twerenbold R, Reiter M, Hochholzer W, Burkhalter $\mathrm{H}$, et al. Utility of absolute and relative changes in cardiac troponin concentrations in the early diagnosis of acute myocardial infarction. Circulation 2011; 124(2):136-45. doi: 10.1161/CIRCULATIONAHA.111.023937. 